\title{
ACOLHIMENTO COM CLASSIFICACCÃO DE RISCO EM UM HOSPITAL DA REDE PÚBLICA: PERCEPÇÃO DOS USUÁRIOS
}

\section{Elaine Cristina Santos Garcia Moreno}

Enfermeira. Universidade Federal de Sergipe, Lagarto (SE), Brasil.

\section{Flavia Maria dos Santos}

Enfermeira. Universidade Federal de Sergipe, Lagarto (SE), Brasil

\section{Déborah Danielle Tertuliano Marinho} fermagem, Universidade Federal de Sergipe, Lagarto (SE), Brasil.

\section{José Marcos de Jesus Santos}

Discente do curso Enfermagem, Universidade Federal de Sergipe, Lagarto (SE), Brasil.

\section{Jéssica Oliveira da Cunha}

Enfermeira. Universidade Federal de Sergipe, Lagarto (SE), Brasil
Enfermeira, Mestre, Docente do Departamento de En-

RESUMO: O objetivo deste estudo foi conhecer a percepção dos usuários quanto ao acolhimento com classificação de risco em um hospital público. Trata-se de um estudo transversal e quantitativo, com abordagens descritiva e analítica, realizado por meio de entrevista com 80 usuários de um hospital público em Itabaiana, Sergipe. Os resultados mostraram que, dentre os participantes que já haviam passado por atendimento médico na unidade hospitalar $(\mathrm{n}=72)$, somente $19,4 \%$ $(n=14)$ referiram conhecimento sobre o que seria a classificação de risco. O tempo de espera $\geq 1$ hora foi referido por $16,3 \%(n=13)$ dos participantes. A maioria foi atendida por técnicos de enfermagem $(65 \% ; n=52)$ e apenas 3,8\% $(n=3)$ disseram ter recebido informação sobre a classificação de risco recebida. Ainda assim, muitos usuários mostraram-se satisfeitos com este atendimento. Concluiu-se que os usuários desconhecem o significado da classificação de risco na priorização dos atendimentos à saúde.

PALAVRAS-CHAVE: Acolhimento; Hospitais; Cuidados de enfermagem.

\section{RISK-CLASSIFIED ADMITTANCE IN A GOVERNMENT- RUN HOSPITAL: CLIENTS' PERCEPTION}

\begin{abstract}
Clients' perception with regard to risk-classified admittance in a public hospital is analyzed. Current transversal, quantitative, descriptive and analytic study comprised interviews with 80 clients of a government-run hospital in Itabaiana SE Brazil. Results showed that, out of the participants $(n=72)$ who were attended to at the hospital, only $19.4 \%(n=14)$ knew anything about risk classification. Waiting time $\geq 1$ hour was referred to by $16.3 \%(n=13)$ of participants. Most were attended by nursing technicians $(65 \% ; n=52)$ and a mere $3.8 \%$ $(n=3)$ alleged to have received information on risk classification. However, most clients were satisfied with attendance. Results show that clients do not know anything about risk classification within the priority of health attendance.
\end{abstract}

KEY WORDS: Admittance; Hospitals; Nursing care.

\section{INTRODUÇÃO}

No Brasil, o Ministério da Saúde tem priorizado a humanizainício do século XXI, sendo o acolhimento com classificação de risco uma das principais metodologias preconizadas para esta finalidade ${ }^{1}$. 
O acolhimento consiste na recepção e avaliação do usuário a partir de sua chegada ao serviço de saúde, responsabilizando-se integralmente por ele e ouvindo suas queixas e preocupações ${ }^{2}$. A classificação de risco, por sua vez, visa priorizar o atendimento de acordo com a gravidade clínica do indivíduo, buscando condições de risco, organizando o trabalho, proporcionando orientações à clientela quanto à necessidade do tempo de espera e reduzindo a ansiedade gerada pelo desconhecimento de todo esse processo por parte da população em geral ${ }^{3}$.

Ressalta-se que nas Unidades de Urgência, a classificação de pacientes começou a ter legitimidade a partir da portaria MS/GM n ${ }^{0} 2.048 / 2002$, que regulamentou o funcionamento da urgência/emergência em todo território nacional e estabelece, como ferramenta de gestão e organização do serviço, o acolhimento com classificação de riscó ${ }^{4}$. Ademais, o Ministério da Saúde lançou, em 2004, a Política Nacional de Humanização (PNH) ${ }^{5}$ e, em 2003, o Programa de Qualificação da Gestão do SUS (QualiSUS) ${ }^{6}$. A PNH trouxe, entre outros avanços, a Diretriz do Acolhimento com Classificação de Risco, que constitui um marco no modo de atenção aos usuários do Sistema Único de Saúde (SUS). O QualiSUS, por sua vez, apresentou o investimento necessário para a efetiva implementação desta classificação por meio de protocolo validado ${ }^{5,7}$.

Os protocolos mais utilizados para classificação de risco, visando otimizar a decisão dos profissionais de saúde na priorização de atendimento dos usuários nos serviços de urgência/emergência, em nível mundial, são: o Australian Triage Scale (ATS(C), Canadian Triage Acuity Scale (CTAS $\odot)$, Emergency Severity Index (ESI C) e o Manchester Triage System (MTS(C). Todas essas escalas organizam o atendimento em cinco níveis de prioridade: emergente, muito urgente, urgente, pouco urgente e não urgente ${ }^{8}$. Pontua-se que, de acordo com a resolução $\mathrm{n}^{\mathrm{o}}$ 423/2012 do Conselho Federal de Enfermagem, a classificação de risco e a priorização da assistência em serviços de urgência é privativa do enfermeiro, observadas as disposições legais da profissão?.

É importante destacar que a realização dessa classificação não é uma atividade tão simples quanto parece, uma vez que depende tanto das habilidades e competências profissionais dos enfermeiros como também de fatores externos e subjetivos relacionados ao atendimento, tais como o ambiente de trabalho, o relacionamento interpessoal e a comunicação estabelecida entre profissional e paciente ${ }^{10}$. Isso justifica a necessidade de se realizar capacitações dos profissionais responsáveis pela classificação, pois em um estudo nacional foi evidenciado que, quanto maior a qualificação do profissional, mais assertivas são as classificações de risco atribuídas por ele ${ }^{11}$.

Ainda nesse sentido, uma pesquisa realizada com 314 profissionais de saúde da área de emergência mostrou que $\mathbf{7 5 , 5 \%}$ deles avaliaram como precário o processo de classificação de risco nas unidades onde atuam ${ }^{12}$. Trata-se de um problema comum nos serviços públicos de saúde do país, nos quais são presenciadas grandes filas e disputas da clientela por um rápido atendimento de saúde, principalmente pela falta de critérios de gravidade e por ser levada em consideração apenas a hora de chegada dos usuários. Essa não distinção de riscos ou graus de sofrimento faz com que alguns casos sejam agravados na fila, inclusive com possiblidade de mortes pelo não atendimento em tempo oportuno ${ }^{13}$.

Dessa forma, tendo em vista a importância de se avaliar a qualidade do atendimento para classificação de risco, visando à identificação das potencialidades e também sugerindo soluções às possíveis fragilidades que possam ser encontradas, o presente estudo objetivou conhecer a percepção dos usuários quanto à classificação de risco em um hospital público de Itabaiana, Sergipe.

\section{METODOLOGIA}

Trata-se de um estudo transversal e quantitativo, com abordagens descritiva e analítica, realizado por meio de entrevista com 80 usuários do Sistema Único de Saúde (SUS) em um hospital público de atenção secundária de Itabaiana, Sergipe. Segundo dados cedidos pela gerência do referido hospital, o Acolhimento com Classificação de Risco foi implantado em 2013, baseado na Política Nacional de Humanização/Protocolo de Manchester, utilizando-se das quatro cores do documento: vermelho, amarelo, azul e verde.

O cálculo amostral foi realizado considerando um nível de confiança de 95\% e de erro máximo permi- 
tido de 5\%. Utilizou-se da amostragem aleatória simples para alcance do número amostral, por meio da qual os usuários tiveram igual probabilidade de participar do trabalho. Como critério de inclusão adotou-se usuários de ambos os sexos com idade entre 18 e 80 anos. Foram excluídos aqueles com quaisquer distúrbios cognitivos e que foram classificados nas áreas amarela e vermelha.

A coleta de dados ocorreu entre os meses de janeiro e fevereiro de 2017 por meio de entrevista estruturada com questões relacionadas à condição sociodemográfica e às características do atendimento para classificação de risco. Todos os usuários que aceitaram participar do estudo foram abordados nas áreas azul e verde do hospital, em dois momentos: logo após passarem pelo acolhimento com classificação de risco e depois do atendimento médico ou administração da medicação.

Os dados obtidos foram tabulados no Microsoft Office Excel (2010) e importados para o software IBM ${ }^{\circledR}$ SPSS - Statistical Package for the Social Sciences, versão 20.0, no qual foram analisados. Para a análise estatística, utilizaram-se as técnicas univariada e bivariada para obtenção da distribuição dos valores das frequências absoluta e relativa. Foi utilizado o teste Qui-quadrado para investigar as associações entre as variáveis categóricas e o Exato de Fisher para as categorias com células de baixa frequência, sendo considerado, em ambos os casos, um nível de significância $<0,05$.

Este estudo foi aprovado pelo Comitê de Ética em Pesquisa da Universidade Federal de Sergipe, com o seguinte CAAE: 62430816.1.00005546 e parecer número 1.875.666. Os pesquisadores seguiram as diretrizes e normas regulamentadoras preconizadas nas resoluções $n^{\circ} 466 / 12$ e no 510/16 do Conselho Nacional de Saúde sobre as pesquisas envolvendo seres humanos, por meio da aceitação do Termo de Consentimento Livre Esclarecido para os usuários.

\section{RESULTADOS}

Os 80 usuários elegíveis e aleatoriamente selecionados para participar deste estudo foram entrevistados, sem perdas ou desistências durante a pesquisa.

A média de idade dos participantes foi de 36,9 anos (desvio-padrão $=14,3$ ), sendo 18 a mínima e 76 a máxima. A maioria era do sexo feminino (60\%), possuía algum rendimento mensal $(76,3 \%)$ e $43,8 \%$ viviam com companheiro à época da pesquisa. A cor da pele parda foi a mais referida $(68,8 \%)$, com negros e amarelos correspondendo a uma proporção muito pequena do total da amostra (10\% e 3,8\%, respectivamente). Quanto à escolaridade, $45 \%$ possuíam o ensino fundamental completo ou incompleto (Tabela 1).

Tabela 1. Caracterização sociodemográfica de usuários das áreas azul e verde de um hospital público de atenção secundária $(\mathrm{n}=80)$. Itabaiana, Sergipe, Brasil, 2017

\begin{tabular}{|c|c|c|}
\hline Características sociodemográficas & $\mathbf{N}$ & $\%$ \\
\hline \multicolumn{3}{|l|}{ Sexo } \\
\hline Feminino & 48 & 60 \\
\hline Masculino & 30 & 37,5 \\
\hline Sem resposta & 2 & 2,5 \\
\hline \multicolumn{3}{|l|}{ Idade } \\
\hline$<20$ anos & 8 & 10 \\
\hline 21 a 40 anos & 40 & 50 \\
\hline 41 a 60 anos & 27 & 33,7 \\
\hline$>60$ anos & 5 & 6,3 \\
\hline \multicolumn{3}{|l|}{ Cor da pele } \\
\hline Branco & 14 & 17,4 \\
\hline Pardo & 55 & 68,8 \\
\hline Amarelo & 3 & 3,8 \\
\hline Negro & 8 & 10 \\
\hline \multicolumn{3}{|l|}{ Situação conjugal } \\
\hline Com companheiro & 35 & 43,8 \\
\hline Sem companheiro & 45 & 56,2 \\
\hline \multicolumn{3}{|l|}{ Escolaridade } \\
\hline Não estudou & 3 & 3,8 \\
\hline $\begin{array}{l}\text { Fundamental completo ou incom- } \\
\text { pleto }\end{array}$ & 36 & 45 \\
\hline Médio completo ou incompleto & 34 & 42,5 \\
\hline Superior completo ou incompleto & 5 & 6,2 \\
\hline Sem resposta & 2 & 2,5 \\
\hline \multicolumn{3}{|l|}{ Renda familiar } \\
\hline$<1$ salário mínimo & 38 & 47,5 \\
\hline 1 a 2 salários mínimos & 15 & 18,7 \\
\hline 2 a 3 salários mínimos & 5 & 6,3 \\
\hline > 3 salários mínimos & 3 & 3,8 \\
\hline Sem rendimento & 19 & 23,7 \\
\hline
\end{tabular}

Nota: N (frequência absoluta), \% (frequência relativa). 
Quase a totalidade da amostra já havia passado por atendimento médico na unidade hospitalar onde o presente estudo foi realizado ( $90 \%$ ), e mesmo assim, apenas $19,4 \%$ referiram conhecimento sobre o que seria a classificação de risco (dados não apresentados em tabela).

O tempo de espera $\geq 1$ hora para atendimento para classificação de risco foi referido por 16,3\% (n=13) dos usuários. A maioria das classificações foi realizada pelos técnicos de enfermagem (65\%) e somente 3,8\% dos participantes foram informados sobre a classificação de risco recebida, bem como nenhum deles tiveram explicação clara do motivo de realizá-la. Ressalta-se que somente $28,7 \%$ dos participantes consideraram que seu problema foi totalmente resolvido e $10 \%$ sugeriram melhorias no atendimento da unidade hospitalar estudada (Tabela 2).

As sugestões foram: "melhorar o atendimento" (37,5\%), "dar mais informações no atendimento" (25\%), "ter mais médicos para atender" (12,5\%), "ter mais agilidade para atender" $(12,5 \%)$ e "diminuir o tempo de espera" (12,5\%) (dados não apresentados em tabelas).

Todavia, ainda assim, mais da metade dos usuários consideraram a qualidade do atendimento para classificação de risco como "boa" (60\%) e 8,7\% (n=7) como "excelente". A maioria referiu satisfação com o tempo de espera, seja da sala de espera para a sala de acolhimento com classificação de risco $(71,2 \%)$ ou desta para o atendimento médico (51,3\%). Quanto à oferta de meios de entretenimento durante o tempo de espera, $57,5 \%$ se dispuseram de televisão e $6,3 \%$ de revistas, livros ou jornais (Tabela 2).

Tabela 2. Características do atendimento oferecido aos usuários das áreas azul e verde de um hospital público de atenção secundária $(\mathrm{N}=80)$. Itabaiana, Sergipe, Brasil, 2017

(Continua)

\begin{tabular}{l|c|c}
\hline Características do atendimento & N & $\%$ \\
\hline $\begin{array}{l}\text { Tempo de espera para atendimento para clas- } \\
\text { sificação de risco }\end{array}$ & & \\
\hline Imediatamente & 27 & 33,7 \\
\hline$<1$ hora & 39 & 48,7 \\
\hline 1 a 2 horas & 8 & 10 \\
\hline$\geq 3$ horas & 5 & 6,3 \\
\hline Sem resposta & 1 & 1,3 \\
\hline $\begin{array}{l}\text { Orientação sobre o motivo do tempo de espe- } \\
\text { ra para classificação }\end{array}$ & & \\
\hline
\end{tabular}

(Conclusão)

\begin{tabular}{|c|c|c|}
\hline Sim & 2 & 2,5 \\
\hline Não & 78 & 97,5 \\
\hline \multicolumn{3}{|l|}{$\begin{array}{l}\text { Categoria profissional que realizou o acolhi- } \\
\text { mento com classificação de risco }\end{array}$} \\
\hline Técnico de enfermagem & 52 & 65 \\
\hline Enfermeiro & 24 & 30 \\
\hline Sem resposta & 4 & 5 \\
\hline \multicolumn{3}{|l|}{$\begin{array}{l}\text { Foi informado sobre a classificação de risco } \\
\text { recebida }\end{array}$} \\
\hline Sim & 3 & 3,8 \\
\hline Não & 77 & 96,2 \\
\hline \multicolumn{3}{|l|}{$\begin{array}{l}\text { Qualidade do atendimento na classificação de } \\
\text { risco }\end{array}$} \\
\hline Excelente & 7 & 8,7 \\
\hline Boa & 48 & 60 \\
\hline Regular & 19 & 23 \\
\hline Ruim & 5 & 6,3 \\
\hline Sem resposta & 1 & 1,3 \\
\hline \multicolumn{3}{|l|}{$\begin{array}{l}\text { Satisfação com o tempo de espera da sala de } \\
\text { espera para a sala de acolhimento com classi- } \\
\text { ficação de risco }\end{array}$} \\
\hline Muito satisfeito & 4 & 5 \\
\hline Satisfeito & 53 & 66,2 \\
\hline Insatisfeito & 19 & 23,7 \\
\hline Muito insatisfeito & 3 & 3,8 \\
\hline Sem resposta & 1 & 1,3 \\
\hline \multicolumn{3}{|l|}{$\begin{array}{l}\text { Satisfação com o tempo de espera da sala de } \\
\text { acolhimento com classificação de risco para o } \\
\text { atendimento médico }\end{array}$} \\
\hline Muito satisfeito & 5 & 6,3 \\
\hline Satisfeito & 36 & 45 \\
\hline Insatisfeito & 23 & 28,7 \\
\hline Muito insatisfeito & 3 & 3,8 \\
\hline Sem resposta & 13 & 16,2 \\
\hline \multicolumn{3}{|l|}{$\begin{array}{l}\text { Serviços de entretenimento ofertados durante } \\
\text { o tempo de espera }\end{array}$} \\
\hline Televisão & 46 & 57,5 \\
\hline Música & 0 & 0 \\
\hline Revistas, livros ou jornais & 5 & 6,3 \\
\hline Nenhum entretenimento & 29 & 36,2 \\
\hline \multicolumn{3}{|l|}{$\begin{array}{l}\text { Resolução do problema que motivou a procu- } \\
\text { ra à unidade hospitalar }\end{array}$} \\
\hline Sim (totalmente) & 23 & 28,7 \\
\hline Sim (parcialmente) & 52 & 65 \\
\hline Não foi resolvido & 3 & 3,8 \\
\hline Sem resposta & 2 & 2,5 \\
\hline
\end{tabular}

Nota: N (frequência absoluta), \% (frequência relativa). 
Na avaliação da associação entre as características do atendimento para classificação de risco e a categoria profissional responsável por sua condução, os resultados descritivos mostraram que os maiores percentuais de atendimentos considerados pelos usuários como bons ou excelentes estão mais associados aos técnicos de enfermagem. Enquanto o tempo de espera reduzido, a satisfação com esse tempo de espera e a resolução do problema que motivou a procura à unidade hospitalar estão mais associados aos atendimentos realizados por enfermeiros. Entretanto, vale ressaltar que não foi identificada evidência estatística de associação entre nenhuma destas variáveis supracitadas $(\mathrm{p}>0,05)$ (Tabela 3$)$.

Tabela 3. Associação entre as características do atendimento para classificação de risco e a categoria profissional responsável $(\mathrm{n}=80)$, Itabaiana, SE, 2017

\begin{tabular}{|c|c|c|c|}
\hline \multirow{2}{*}{$\begin{array}{l}\text { Variáveis relacionadas } \\
\text { ao atendimento para } \\
\text { classificação de risco }\end{array}$} & \multicolumn{2}{|c|}{$\begin{array}{c}\text { Categoria } \\
\text { profissional } \\
\text { que realizou a } \\
\text { classificação de } \\
\text { risco }\end{array}$} & \multirow[t]{2}{*}{$\mathbf{P}$} \\
\hline & $\begin{array}{c}\text { Técnico } \\
\text { N (\%) }\end{array}$ & $\begin{array}{l}\text { Enfer- } \\
\text { meiro } \\
\mathrm{N}(\%)\end{array}$ & \\
\hline Qualidade do atendimento & & & 0,607 \\
\hline Boa ou excelente & $37(71,2)$ & $15(65,2)$ & \\
\hline Regular ou ruim & $15(28,8)$ & $8(34,8)$ & \\
\hline $\begin{array}{l}\text { Conhecimento sobre a classi- } \\
\text { ficação recebida }\end{array}$ & & & $0,465^{*}$ \\
\hline Sabe & $2(3,8)$ & $0(0)$ & \\
\hline Não sabe & $50(96,2)$ & $24(100)$ & \\
\hline $\begin{array}{l}\text { Tempo de espera para classi- } \\
\text { ficação }\end{array}$ & & & $0,762 *$ \\
\hline $\begin{array}{l}\text { Igual ou menor que } 30 \text { minu- } \\
\text { tos }\end{array}$ & $40(78,4)$ & $20(83,3)$ & \\
\hline Maior que 30 minutos & $11(21,6)$ & $4(16,7)$ & \\
\hline $\begin{array}{l}\text { Satisfação com o tempo de } \\
\text { espera }\end{array}$ & & & 0,691 \\
\hline Satisfeito ou muito satisfeito & $36(70,6)$ & $18(75)$ & \\
\hline $\begin{array}{l}\text { Insatisfeito ou muito insatis- } \\
\text { feito }\end{array}$ & $15(29,4)$ & $6(25)$ & \\
\hline $\begin{array}{l}\text { Resolução do problema que } \\
\text { motivou a procura à unidade } \\
\text { hospitalar }\end{array}$ & & & $0,109 *$ \\
\hline Parcialmente ou não resolvido & $32(64)$ & $20(83,3)$ & \\
\hline Totalmente resolvido & $18(36)$ & $4(16,7)$ & \\
\hline $\begin{array}{l}\text { Notas: }{ }^{1} \mathrm{p}=\text { valor de } \mathrm{p} ; \\
{ }^{*} \text { Resultado do teste Exato de Fish } \\
\text { N (frequência absoluta), \% (frequ }\end{array}$ & $\begin{array}{l}\text { r devido } \\
\text { ncia rela }\end{array}$ & $\begin{array}{l}\text { baixa frequ } \\
\text { a). }\end{array}$ & cia. \\
\hline
\end{tabular}

Em relação à associação dos dados sociodemográficos e as características do atendimento para classificação de risco, observou-se que os aspectos positivos do atendimento estão mais relacionados aos usuários do sexo feminino, com idade $\leq 40$ anos, de cor parda e com renda mensal fixa. Entretanto, de modo semelhante, não foi identificada evidência estatística de associação entre nenhuma destas variáveis $(\mathrm{p}>0,05)$ (Tabela 4$)$.

A única exceção de significância estatística diz respeito à associação entre as variáveis renda mensal e satisfação do usuário com o tempo de espera para classificação, cujos resultados mostraram que, dentre os usuários com rendimento mensal fixo, $87,7 \%$ referiram satisfação com o tempo de espera da chegada à unidade até o atendimento para classificação de risco $(\mathrm{p}<0,05)$ (Tabela 4). 
Tabela 4. Associação entre as variáveis sociodemográficas e as características do atendimento para classificação de risco ( $\mathrm{n}=80)$, Itabaiana, SE, 2017

\begin{tabular}{|c|c|c|c|c|c|c|c|c|}
\hline \multirow[b]{3}{*}{ Variáveis } & \multicolumn{8}{|c|}{ Características do atendimento para classificação de risco } \\
\hline & $\begin{array}{c}\text { Qualidade } \\
\text { do atendimento }\end{array}$ & & $\begin{array}{l}\text { Informado sobre a } \\
\text { classificação recebida }\end{array}$ & & $\begin{array}{l}\text { Satisfeito com o } \\
\text { tempo de espera }\end{array}$ & & $\begin{array}{l}\text { Problema } \\
\text { resolvido }\end{array}$ & \\
\hline & $\begin{array}{l}\text { Boa ou } \\
\text { excelente }\end{array}$ & $\mathbf{P}$ & Sim & $\mathbf{P}$ & Sim & $\mathbf{P}$ & Sim & $\mathbf{P}$ \\
\hline Sexo & $\%$ & & $\%$ & & $\%$ & & $\%$ & \\
\hline Feminino & 62,3 & 0,74 & 100 & 0,52 & 56,4 & 0,18 & 62,3 & 0,80 \\
\hline $\begin{array}{l}\text { Masculino } \\
\text { Idade }\end{array}$ & 37,7 & 0 & & $\stackrel{43,6}{1}$ & 37,7 & 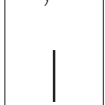 & & \\
\hline $\begin{array}{l}\leq 40 \text { anos } \\
\geq 41 \text { anos }\end{array}$ & $\begin{array}{l}54,5 \\
45,5\end{array}$ & $\begin{array}{l}0,08 \\
33,3\end{array}$ & 66,7 & $\begin{array}{l}0,65 \\
43,9\end{array}$ & $\begin{array}{l}56,1 \\
47,3\end{array}$ & 0,32 & 52,7 & 0,08 \\
\hline Cor da pele & & & & & & & & \\
\hline Branco & 20 & $0,54^{*}$ & 33,3 & $0,49 *$ & 20 & $054 *$ & 12,8 & $000 *$ \\
\hline Pardo & 80 & 66,7 & & 80 & 87,2 & 0,54 & & 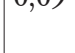 \\
\hline Situação conjugal & & & & & & & & \\
\hline Com companheiro & 43,6 & 0,85 & 66,7 & $0,57^{*}$ & 47,4 & & 45,5 & 060 \\
\hline Sem companheiro & 56,4 & 33,3 & & 52,6 & 54,5 & 0,21 & & 0,00 \\
\hline Escolaridade & & & & & & & & \\
\hline $\begin{array}{l}\text { Fundamental completo } \\
\text { ou incompleto }\end{array}$ & 51 & 0,94 & 0 & $0,23^{*}$ & 54 & 060 & 51 & 2 \\
\hline $\begin{array}{l}\text { Médio completo ou in- } \\
\text { completo }\end{array}$ & 49 & 100 & & 46 & 49 & 0,02 & & 0,93 \\
\hline Renda mensal & & | & & 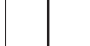 & & & & \\
\hline Possui & 80 & 0,37 & 100 & $0,43^{*}$ & 87,7 & & 73,9 & \\
\hline Não possui & 20 & 0 & & 12,3 & 26,1 & $<0,01$ & & 0,81 \\
\hline
\end{tabular}

Notas: ${ }^{1} \mathrm{p}=$ valor de $\mathrm{p}$

${ }^{2 *}$ Resultado do teste Exato de Fisher devido à baixa frequência.

${ }^{3} \mathrm{~N}$ (frequência absoluta), \% (frequência relativa).

${ }^{4}$ Diferença estatisticamente significativa em negrito $(p<0,05)$.

${ }^{5}$ Excluíram-se os participantes que não responderam cada variável de forma específica.

\section{DISCUSSÃO}

Acredita-se que o conhecimento do perfil sociodemográfico da clientela atendida em um determinado serviço de saúde é de fundamental importância para o estabelecimento de prioridades e para o planejamento de ações mais adequadas à realidade dos usuários. ${ }^{14}$ Nesse sentido, observou-se no presente estudo que a maioria dos participantes era do sexo feminino (60\%), possuía algum rendimento mensal (76,3\%) e 43,8\% viviam com companheiro(a). A cor da pele parda foi a mais referida
$(68,8 \%)$ e, quanto à escolaridade, $45 \%$ possuíam o ensino fundamental à época da pesquisa.

No que se refere às características do atendimento, foi constatado que a maioria dos usuários já havia passado pelo acolhimento com classificação de risco e consulta médica na unidade hospitalar onde o estudo foi realizado (90\%), e mesmo assim, somente $19,4 \%$ deles referiram conhecimento sobre o que seria esta classificação. Esse resultado corrobora com a evidência de outro estudo nacional que percebeu a necessidade de maior divulgação da classificação de risco para a sociedade em 
geral, uma vez que quando são classificados como de menor prioridade para o atendimento, os usuários costumam referir insegurança e insatisfação com a assistência recebida ${ }^{15}$

Ademais, como fator agravante dessa insatisfação por parte da população, deve-se citar ainda o fato dos serviços de urgência e emergência serem cada vez mais procurados, sobretudo por àqueles que teriam seu problema resolvido na atenção primária, e com isso, consequentemente, observa-se uma demanda cada vez maior nestes locais, o que gera superlotação. Assim, comumente sobressai indissociavelmente, a precariedade dos serviços, nos quais não raramente, nos deparamos com corredores aglomerados de pacientes em macas sem colchões, sem privacidade, conforto ou segurança, à espera de atendimento e, ainda, vulneráveis a infecções ${ }^{16,17}$.

O tempo de espera $\geq 1$ hora para atendimento para classificação de risco foi referido por $16,3 \%$ dos usuários, fato semelhante ao encontrado em outro trabalho com delineamento semelhante ${ }^{18}$. Sabe-se que a rapidez para o atendimento é um fator importante à manutenção da vida e à prevenção de sequelas. Todavia, para isso, é necessária a organização do processo de trabalho da equipe multiprofissional, pois se trata de um elemento que intervém na qualidade da assistência prestada e reflete diretamente nos resultados assistenciais ${ }^{15}$.

Ademais, embora exista a recomendação de que a classificação de risco seja realizada por enfermeiros, uma vez que essa categoria possui uma linguagem clínica orientada para os sinais e sintomas e uma relação empática fundamental para o objetivo do processo de atendimento ${ }^{19}$, observou-se que, no presente estudo, a maioria das classificações foi realizada pelos técnicos de enfermagem (65\%). A cautela quanto ao profissional que realiza esta classificação diz respeito à grande responsabilidade do mesmo em atuar como regulador na porta de entrada do serviço de saúde, tendo o poder de decisão sobre qual paciente deve ter seu atendimento priorizado pelos demais profissionais da unidade ${ }^{20}$.

Outra importante deficiência relacionada ao atendimento para classificação de risco entre os participantes deste trabalho diz respeito à informação ao usuário quanto à classificação de risco que recebeu, pois apenas 3,8\% da amostra total referiram o recebimento de tal relato por parte do profissional de saúde que lhe atendeu. Nesse sentido, reforça-se que é direito do paciente conhecer seu grau de urgência, tempo de espera e prioridade no atendimento ainda na sala de classificação de risco ${ }^{21}$.

Ressalta-se também que, apesar de não haver evidência estatística de associação, os resultados descritivos deste trabalho mostraram que, na avaliação da associação entre as características do atendimento para classificação de risco e a categoria profissional responsável por sua condução, os maiores percentuais de satisfação dos usuários com o tempo de espera e de resolução do problema que motivou a procura à unidade hospitalar estão mais associados aos atendimentos realizados por enfermeiros. Sabe-se que esta categoria profissional é um referencial na aplicação da classificação de risco, sendo qualificada para isso desde sua formação, direcionando a avaliação de forma integral ao paciente e não apenas ao seu diagnóstico clínico ${ }^{22}$.

Quanto à oferta de meios de entretenimento para os usuários durante o tempo de espera para atendimento médico, verificou-se que $36,2 \%$ dos participantes não se dispuseram de nenhuma distração na unidade hospitalar estudada. Nesse sentido, é importante que sejam realizadas ações de educação em saúde para preenchimento produtivo desse tempo ocioso e também para promoção de um maior contato entre o usuário e a equipe de saúde atuante no serviço ${ }^{23}$.

Em relação à resolutividade, somente $28,7 \%$ dos usuários consideraram que seu problema foi totalmente resolvido com o atendimento médico recebido. Resta pontuar que este percentual se encontra abaixo do identificado em um estudo realizado em Fortaleza, Ceará, no qual $65,4 \%$ da amostra referiu a resolução do problema que motivou a procura ao serviço de saúde ${ }^{18}$. Trata-se, portanto, de um indicador negativo importante, já que contradiz um dos princípios gerais do acolhimento: o compromisso de fornecer respostas às necessidades de saúde trazidas pelos usuários ${ }^{24}$.

Por fim, faz-se necessário discutir que, mesmo diante das deficiências supracitadas, no que se refere às características do atendimento para classificação de risco na unidade hospitalar estudada, mais da metade dos participantes classificou a qualidade desse atendimento, de 
forma geral, como "boa" (60\%). Nesse sentido, pontuase o fato de ter havido predomínio de participantes com baixo nível socioeconômico no presente estudo, uma vez que dados observados em pesquisas similares indicam que este é um fator que influencia na avaliação da satisfação de usuários de instituições de saúde ${ }^{10,25}$.

\section{CONCLUSÃO}

O presente estudo permitiu conhecer a percepção dos usuários quanto ao atendimento para classificação de risco em um serviço público de saúde, com resultados que corroboram com os achados da literatura científica disponível. Foi evidenciado o desconhecimento do significado da classificação de risco para priorização dos atendimentos à saúde por parte da população estudada, bem como se percebeu o conformismo da maioria frente aos problemas funcionais que merecem adequação.

No que concerne à Classificação de Risco, observou-se discordância com o que é preconizado pela Política Nacional de Humanização, visto que o profissional mais indicado para este atendimento é o enfermeiro.

Dessa forma, destaca-se a necessidade de maior divulgação dos preceitos relacionados à classificação de risco à sociedade em geral, sobretudo no momento do acolhimento no Sistema Único de Saúde, tendo em vista que, para isso, não são necessários grandes recursos tecnológicos, apenas sensibilização e responsabilização profissional para com o ser humano de forma integral.

\section{REFERÊNCIAS}

1. Brasil. Ministério da Saúde. Política Nacional de Normalização - Atenção Hospitalar. Brasília, DF: Ministério da Saúde; 2013.

2. Gibaut MAM, Hori LMR, Freitas KS, Mussi FC. Comfort of the patient's family in an Intensive Care Unit related to welcoming. Rev. esc. enferm. USP. 2013;47(5):1114-21.

3. Nishio EA, Franco MTG. Modelo de Gestão em Enfermagem: qualidade assistencial e segurança do paciente. Rio de Janeiro: Elsevier; 2011.
4. Brasil. Portaria ${ }^{0} 2.048$, de 5 de novembro de 2002. Institui o Regulamento Técnico dos Sistemas Estaduais de Urgência e Emergência. Diário Oficial da República Federativa do Brasil, Seção 1, p. 32-54, 2002.

5. Brasil. Ministério da Saúde. HumanizaSUS: Documento base para gestores e trabalhadores do SUS. Brasília, DF: Ministério da Saúde; 2010.

6. Gusmão-Filho FAR, Carvalho EF, Júnior JLAC. Avaliação do grau de implantação do Programa de Qualificação da Atenção Hospitalar de Urgência (Qualisus). Cien Saude Colet. 2010;15(1):1227-38.

7. Brasil. Portaria no 396, de 4 de março de 2011. Institui o Projeto de Formação e Melhoria da Qualidade de Rede de Saúde (Quali-SUS-Rede) e suas diretrizes operacionais gerais. Diario Oficial da União; 2011.

8. Pinto Júnior D, Salgado PO, Chianca TCM. Validade preditiva do Protocolo de Classificação de Risco de Manchester: avaliação da evolução dos pacientes admitidos em um pronto atendimento. Rev Lat Am Enfermagem. 2012;20(6):1-8.

9. Conselho Federal de Enfermagem. Resolução ${ }^{0}$ 423/2012. Brasília, DF: COFEN; 2012.

10. Silva PL, Paiva L, Faria VB, Ohl RIB, Chavaglia SRR. Triage in an adult emergency service: patient satisfaction. Rev Esc Enferm USP. 2016;50(3):427-32.

11. Silva MFN, Oliveira GN, Pergola-Marconato AM, Marconato RS, Bargas EB, Araujo IEM. Protocolo de avaliação e classificação de risco de pacientes em unidade de emergência. Rev Lat Am Enfermagem. 2014;22(2):218-25.

12. Bellucci Júnior JA, Vituri DW, Versa GLGS, Furuya PS, Vidor RC, Matsuda LM. Acolhimento com classificação de risco em serviço hospitalar de emergência: avaliação do processo de atendimento. Rev enferm UERJ. 2015;23(1):82-7.

13. Brasil. Ministério da Saúde. Acolhimento e Classificação de Risco nos Serviços de Urgência. Brasília, DF: Ministério da Saúde; 2009. 
14. Furtado BMASM, Araújo J, Cavalcanti JLP. O perfil da emergência do Hospital da Restauração: uma análise dos possíveis impactos após a municipalização dos serviços de saúde. Rev. bras. epidemiol. 2004;7(3):279-89.

15. Oliveira JLC, Gatti AP, Barreto MS, Bellucci Junior JA, Góes HLF, Matsuda LM. Acolhimento com classificação de risco: percepções de usuários de uma unidade de pronto atendimento. Texto Contexto Enferm. 2017;26(1):1-8.

16. Andrade LM, Martins EC, Caetano JA, Soares E, Beserra EP. Atendimento humanizado nos serviços de emergência hospitalar na percepção do acompanhante. Rev. Eletr. Enf. 2009;11(1):151-7.

17. Brunner e Suddarth. Tratado de Enfermagem médico-cirúrgica. 12ed. Rio de Janeiro: Guanabara Koogan; 2012. v. 2, p. 893-905.

18. Guedes MVC, Henriques ACPT, Lima MMN. Acolhimento em um serviço de emergência: percepção dos usuários. Rev Bras Enferm. 2013;66(1):31-7.

19. Duro CLM, Lima MADS. The nurse's role in Emergency Triage Systems : literature analysis. Online braz $j$ nurs. 2011;9(3):1-12.

20. Souza CC, Diniz AS, Silva LLT, Mata LRF, Chianca TCM. Nurses' perception about risk classification in an emergency service. Invest Educ Enferm. 2013;32(1):78-86.

21. Bellucci Júnior JA, Matsuda LM. Implantação do sistema acolhimento com Classificação e avaliação de risco e uso do fluxograma analisador. Texto Contexto Enferm. 2012;21(1):217-25.

22. Lopes JB. Enfermeiro na classificação de risco em serviços de emergência: revisão integrativa. Monografia. Universidade Federal do Rio Grande do Sul; 2011.

23. Teixeira E, Veloso R. O grupo em sala de espera: território de práticas e representações em saúde. Texto Contexto Enferm. 2006;15(2):320-25.

24. Brasil. Ministério da Saúde. Humaniza SUS: acolhi- mento com avaliação e classificação de risco - um paradigma ético-estético no fazer em saúde. Brasília, DF: Ministério da Saúde; 2004.

25. Paiva SMA, Gomes ELR. Assistência hospitalar: avaliação da satisfação dos usuários durante seu período de internação. Rev Lat Am Enfermagem. 2007;15(5):973-79.

Recebido em: 18/07/2017 Aceito em: 22/02/2018 